\title{
MORITA THEOREMS FOR FUNCTOR CATEGORIES
}

\author{
BY \\ D. C. NEWELL
}

\begin{abstract}
We generalize the Morita theorems to certain functor categories using properties of adjoint functors.
\end{abstract}

Let $\mathscr{A}$ and $\mathscr{B}$ be categories. Recall that $\mathscr{A}$ and $\mathscr{B}$ are said to be equivalent if there are functors $u: \mathscr{A} \rightarrow \mathscr{B}$ and $v: \mathscr{B} \rightarrow \mathscr{A}$ and natural isomorphisms of functors $\eta: I \Rightarrow v u$ and $\varepsilon: u v \Rightarrow I^{\prime}$, where $I$ and $I^{\prime}$ denote the identity functors on $\mathscr{A}$ and $\mathscr{B}$ respectively. When $\mathscr{A}=\mathscr{M}_{R}$ and $\mathscr{B}=\mathscr{M}_{S}$, where $R$ and $S$ are unitary rings and $\mathscr{M}_{R}$ and $\mathscr{M}_{S}$ denote the categories of right $R$-modules and right $S$-modules respectively, the Morita theorems state that the pairs of functors $(v, u), u: \mathscr{A} \rightarrow \mathscr{B}$ and $v: \mathscr{B} \rightarrow \mathscr{A}$, which define an equivalence of categories between $\mathscr{A}$ and $\mathscr{B}$ are in one-to-one correspondence with bimodules ${ }_{R} E_{S}$ which are $R-S$ progenerators, the correspondence being given by isomorphisms $u \cong \bigotimes_{R} E$ and $v \cong \operatorname{Hom}_{S}(E, \quad$ ) (see [2], or [1, Chapter II, §3] for a discussion of these theorems).

In general, the isomorphisms $u \cong \otimes_{R} E$ and $v \cong \operatorname{Hom}_{S}(E, \quad)$ give a one-to-one correspondence between bimodules ${ }_{R} E_{S}$ and adjoint pairs $(v, u)$ from $\mathscr{M}_{S}$ to $\mathscr{M}_{R}$ i.e. functors $v: \mathscr{M}_{S} \rightarrow \mathscr{M}_{R}$ having $u$ as a left adjoint. Since the class of pairs $(v, u)$ which give an equivalence of categories is a subclass of the class of adjoint pairs, one may view the Morita theorems as characterizing the corresponding subclass of bimodules. In [4], we have shown that the class of adjoint pairs between certain functor categories is in one-to-one correspondence with bifunctors in a manner generalizing the above correspondence on module categories. In this paper, we generalize the Morita theorems to characterize those bifunctors which give an equivalence of functor categories.

Our exposition and results parallel [2], but our proofs use properties of adjoint functors and results of [4]. §1 summarizes these properties and results and introduces the machinery for the rest of the paper, $\$ 2$ generalizes the notion of "projectives" and "generators" to bifunctors, $\$ 3$ generalizes the notion of "Morita context" to bifunctors, while $\$ 4$ is devoted to our theorem.

Our results will be stated in the following setting: Ab will denote the category of abelian groups, all of our categories will be preadditive, and all of our functors will be additive. However, it is clear that our results will hold for $\mathscr{V}$-categories, where $\mathscr{V}$ is a closed category satisfying certain "completion" conditions.

Received by the editors June 24, 1969 and, in revised form, July 27, 1971.

AMS 1970 subject classifications. Primary 18A25; Secondary 18G99.

Key words and phrases. Functor categories, equivalence of categories, adjoint functors, bifunctors, small projectives, generators, Morita context.

Copyright (C) 1972, American Mathematical Society 
We shall always assume that we are in a proper set-theoretical setting so that our functor categories are defined.

1. Adjoint functors, bifunctors, and duality. Let $\mathscr{A}$ and $\mathscr{B}$ be two categories, and let $u: \mathscr{A} \rightarrow \mathscr{B}$ and $v: \mathscr{B} \rightarrow \mathscr{A}$ be functors. Then $(v, u)$ is an adjoint pair from $\mathscr{B}$ to $\mathscr{A}$ if $u$ is a left adjoint to $v$ i.e. for each pair of objects $A$ of $\mathscr{A}$ and $B$ of $\mathscr{B}$, there is an isomorphism natural in $A$ and $B$

$$
\theta(A, B): \mathscr{B}(u A, B) \rightarrow \mathscr{A}(A, v B)
$$

(where we use the notation $\mathscr{A}\left(A, A^{\prime}\right)$ to denote the set of morphisms in $\mathscr{A}$ from the object $A$ to the object $A^{\prime}$ ). Let $I_{\mathscr{A}}$ and $I_{\mathscr{B}}$ denote the identity functors on $\mathscr{A}$ and $\mathscr{B}$ respectively. Then if $(v, u)$ is an adjoint pair from $\mathscr{B}$ to $\mathscr{A}$, we may define natural transformations $\eta: I_{\mathscr{A}} \Rightarrow v u$ and $\varepsilon: u v \Rightarrow I_{\mathscr{B}}$ by $\eta_{A}=\theta(A, u A)\left(1_{u A}\right)$ for every object $A$ of $\mathscr{A}$ and $\varepsilon_{B}=\theta^{-1}(v B, B)\left(1_{v B}\right)$ for every object $B$ of $\mathscr{B}$, where $1_{u A}$ and $1_{v B}$ denote the identity morphisms on the objects $u A$ and $u B$ respectively. We shall call $\eta$ and $\varepsilon$ the unit and the counit of the adjoint pair $(v, u)$ respectively. They satisfy the following identities:

$$
u \stackrel{u \eta}{\Longrightarrow} u v u \stackrel{\varepsilon u}{\Longrightarrow} u=1_{u}, \quad v \stackrel{\eta v}{\Longrightarrow} v u v \stackrel{v \varepsilon}{\Longrightarrow} v=1_{v} .
$$

For a proof of this, or any property of adjoint pairs that we use in this paper, we refer the reader to [5].

We shall let $\operatorname{Adj}(\mathscr{B}, \mathscr{A})$ denote the category whose objects are adjoint pairs $(v, u)$ from $\mathscr{B}$ to $\mathscr{A}$, and whose morphisms from an adjoint pair $(v, u)$ to another $\left(v^{\prime}, u^{\prime}\right)$ are natural transformations $u \Rightarrow u^{\prime}$ (these are in one-to-one correspondence with natural transformations $v^{\prime} \Rightarrow v$ ).

We let the reader establish the fact that two functors $u: \mathscr{A} \rightarrow \mathscr{B}$ and $v: \mathscr{B} \rightarrow \mathscr{A}$ define an equivalence of categories if and only if $(v, u)$ is an object of $\operatorname{Adj}(\mathscr{B}, \mathscr{A})$ whose unit and counit are isomorphisms.

For a small (preadditive) category $\mathscr{C}$, let us set down the following notation which we shall use throughout this paper. $\mathscr{C}^{\circ}$ will denote the dual category of $\mathscr{C}$, and for any object $C$ of $\mathscr{C}, \mathscr{C}(C, \quad): \mathscr{C} \rightarrow \mathrm{Ab}$ and $\mathscr{C}(, C): \mathscr{C}^{\circ} \rightarrow \mathrm{Ab}$ will denote the corresponding covariant and contravariant hom-functors. We shall also use $\mathscr{C}$ to denote the bifunctor $\mathscr{C}(, \quad): \mathscr{C}^{\circ} \times \mathscr{C} \rightarrow \mathrm{Ab}$ (given on objects by $\mathscr{C}(, \quad)\left(C, C^{\prime}\right)$ $\left.=\mathscr{C}\left(C, C^{\prime}\right)\right)$ if no confusion develops. [ $\left.\mathscr{C}, \mathrm{Ab}\right]$ will denote the functor category whose objects are (additive) functors from $\mathscr{C}$ to $\mathrm{Ab}$ and whose morphisms are natural transformations. For $F$ and $G$ objects of $[\mathscr{C}, \mathrm{Ab}]$, we shall denote the set of natural transformations from $F$ to $G$ by $[F, G]$.

Let $\mathscr{C}$ and $\mathscr{C}^{\prime}$ be small categories, and let $U: \mathscr{C}^{\circ} \times \mathscr{C}^{\prime} \rightarrow \mathrm{Ab}$ be a bifunctor. In [4], a functor

$$
\otimes_{\mathscr{C}} U:[\mathscr{C}, \mathrm{Ab}] \rightarrow\left[\mathscr{C}^{\prime}, \mathrm{Ab}\right]
$$

is defined, and is characterized by the properties that it is right continuous (i.e. preserves right limits) and, for every object $C$ of $\mathscr{C}, \mathscr{C}(C, \quad) \otimes_{\mathscr{C}} U=U(C, \quad)$. 
Suppose $t:[\mathscr{C}, \mathrm{Ab}] \rightarrow\left[\mathscr{C}^{\prime}, \mathrm{Ab}\right]$ is a functor. Then there is a bifunctor $U_{t}: \mathscr{C}^{\circ} \times \mathscr{C}^{\prime}$ $\rightarrow \mathrm{Ab}$, which we call the bifunctor corresponding to $t$, defined by

$$
U_{t}(A, B)=t(\mathscr{C}(A, \quad))(B) .
$$

This bifunctor has the following property:

THEOREM 1.1. Let $t:[\mathscr{C}, \mathrm{Ab}] \rightarrow\left[\mathscr{C}^{\prime}, \mathrm{Ab}\right]$ be a functor with corresponding bifunctor $U_{t}: \mathscr{C}^{\circ} \times \mathscr{C}^{\prime} \rightarrow \mathrm{Ab}$. Then there is a unique natural transformation

$$
\Psi_{t}: \otimes_{\mathscr{b}} U_{t} \Rightarrow t
$$

for which $\Psi_{t}(\mathscr{C}(A, \quad))$ is the identity on $U_{t}(A, \quad)$ for every object $A$ of $\mathscr{C} . \Psi_{t}$ satisfies the universal property that if $U: \mathscr{C}^{\circ} \times \mathscr{C}^{\prime} \rightarrow \mathrm{Ab}$ is any bifunctor and $\lambda: \quad \otimes_{\mathscr{C}} U \Rightarrow t$ is any natural transformation, then there is a unique natural transformation of bifunctors $\bar{\lambda}: U \Rightarrow U_{t}$ for which the diagram

$\otimes \bar{\lambda}$

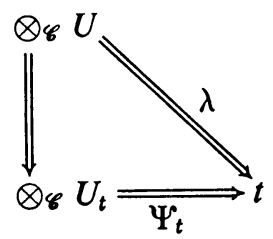

commutes. Furthermore, $\Psi_{t}$ is an isomorphism if, and only if, $t$ is right continuous.

For a proof, one may see [4, 1.2 and 2.1] or supply the proof himself.

For $U: \mathscr{C}^{\circ} \times \mathscr{C}^{\prime} \rightarrow \mathrm{Ab}$ a bifunctor, we define a functor

$$
[U,]_{\mathscr{G}}:\left[\mathscr{C}^{\prime}, \mathrm{Ab}\right] \rightarrow[\mathscr{C}, \mathrm{Ab}]
$$

by $[U, G]_{\mathscr{C}},(A)=[U(A, \quad], G]$ for every functor $G$ in $\left[\mathscr{C}^{\prime}, \mathrm{Ab}\right]$ and for every object $A$ of $\mathscr{C}$. We restate [4, 1.3 and 2.2] in the following:

THEOREM 1.2. If $U: \mathscr{C}^{\circ} \times \mathscr{C}^{\prime} \rightarrow \mathrm{Ab}$ is a bifunctor, then $\Phi(U)=\left([U, \quad]_{\mathscr{C}^{\prime}}, \otimes_{\mathscr{C}} U\right)$ is an adjoint pair from $\left[\mathscr{C}^{\prime}, \mathrm{Ab}\right]$ to $[\mathscr{C}, \mathrm{Ab}]$ (which we shall call the adjoint pair corresponding to $U)$. The functor

$$
\Phi:\left[\mathscr{C}^{\circ} \times \mathscr{C}^{\prime}, \mathrm{Ab}\right] \rightarrow \operatorname{Adj}\left(\left[\mathscr{C}^{\prime}, \mathrm{Ab}\right],[\mathscr{C}, \mathrm{Ab}]\right)
$$

defined by this correspondence is an equivalence of categories.

We remark that the "inverse" of $\Phi$ is the functor that assigns to each adjoint pair $(v, u)$ the bifunctor $U_{u}$ corresponding to $u$.

For bifunctors $U: \mathscr{C}_{1}^{\circ} \times \mathscr{C}_{2} \rightarrow \mathrm{Ab}$ and $V: \mathscr{C}_{2}^{\circ} \times \mathscr{C}_{3} \rightarrow \mathrm{Ab}$, we define a bifunctor

$$
U \otimes \mathscr{C}_{2} V: \mathscr{C}_{1}^{\circ} \times \mathscr{C}_{3} \rightarrow \mathrm{Ab}
$$

by $\left(U \otimes_{\mathscr{C}_{2}} V\right)(A, C)=\left(U(A, \quad) \otimes_{\mathscr{C}_{2}} V\right)(C)$. In [4, 2.3] we have shown the following:

Proposition 1.3. Let $U: \mathscr{C}_{1}^{\circ} \times \mathscr{C}_{2} \rightarrow \mathrm{Ab}$ and $V: \mathscr{C}_{2}^{\circ} \times \mathscr{C}_{3} \rightarrow \mathrm{Ab}$ be bifunctors. Then

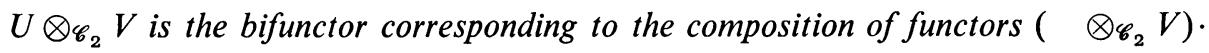
$\left(\otimes_{\mathscr{C}_{1}} U\right)$. 
From this proposition, one easily shows that the tensor product of bifunctors is associative.

Let $U_{1}: \mathscr{C}_{3}^{\circ} \times \mathscr{C}_{2} \rightarrow \mathrm{Ab}$ and $U_{2}: \mathscr{C}_{1}^{\circ} \times \mathscr{C}_{2} \rightarrow \mathrm{Ab}$ be two bifunctors. Then we define a bifunctor

$$
H_{\mathscr{C}_{2}}\left[U_{1}, U_{2}\right]: \mathscr{C}_{1}^{\circ} \times \mathscr{C}_{3} \rightarrow \mathrm{Ab}
$$

by $H_{\mathscr{C}_{2}}\left[U_{1}, U_{2}\right](A, C)=\left[U_{1}(C, \quad), U_{2}(A, \quad)\right]$ for every object $A$ of $\mathscr{C}_{1}$ and $C$ of $\mathscr{C}_{3}$ (note that $U_{1}(C, \quad)$ and $U_{2}(A, \quad)$ are functors in [ $\left.\left.\mathscr{C}_{2}, \mathrm{Ab}\right]\right)$. Similarly, for bifunctors $V_{1}: \mathscr{C}_{1}^{\circ} \times \mathscr{C}_{2} \rightarrow \mathrm{Ab}$ and $V_{2}: \mathscr{C}_{1}^{\circ} \times \mathscr{C}_{3} \rightarrow \mathrm{Ab}$, we define a bifunctor

$$
H^{\mathscr{C}_{1}}\left[V_{1}, V_{2}\right]: \mathscr{C}_{2}^{\circ} \times \mathscr{C}_{3} \rightarrow \mathrm{Ab}
$$

by $H^{\mathscr{C}_{1}}\left[V_{1}, V_{2}\right](B, C)=\left[V_{1}(, B), V_{2}(, C)\right]$.

In the following, let us fix a bifunctor $U: \mathscr{C}^{\circ} \times \mathscr{C}^{\prime} \rightarrow \mathrm{Ab}$, its corresponding adjoint pair $(v, u)$ in $\operatorname{Adj}\left(\left[\mathscr{C}^{\prime}, \mathrm{Ab}\right],[\mathscr{C}, \mathrm{Ab}]\right)$ with unit $\eta: I \Rightarrow v u$ and counit $\varepsilon: u v \Rightarrow I^{\prime}$ ( $I$ and $I^{\prime}$ being the identity functors on $[\mathscr{C}, \mathrm{Ab}]$ and $\left[\mathscr{C}^{\prime}, \mathrm{Ab}\right]$ respectively), recalling, from 1.2, that $\otimes_{\mathscr{C}} U=u$ and $[U,]_{\mathscr{C}}=v$.

We may define a natural transformation of bifunctors

$$
\alpha_{U}: \mathscr{C} \rightarrow H_{\mathscr{C}}[U, U]
$$

by $\alpha_{U}\left(C_{1}, C_{2}\right)(f)=U(f, \quad)$ for every pair of objects $C_{1}, C_{2}$ of $\mathscr{C}$ and for every morphism $f: C_{1} \rightarrow C_{2}$ in $\mathscr{C} . \alpha_{U}$ may be described in another way as follows. One sees by 1.1 that the bifunctor $\mathscr{C}$ corresponds to $I$, so that one has an isomorphism $\Psi: \quad \otimes_{\mathscr{C}} \mathscr{C} \cong I$. Also, one easily checks that for any pair of objects $C$ of $\mathscr{C}$ and $C^{\prime}$ of $\mathscr{C}^{\prime}, H_{\mathscr{C}},[U, U]\left(C, C^{\prime}\right) \cong(v u(\mathscr{C}(C))),\left(C^{\prime}\right)$, so that $H_{\mathscr{C}},[U, U]$ is the bifunctor corresponding to $v u$, and consequently by 1.1 we have a natural transformation

$$
\Psi_{v u}: \quad \otimes_{\mathscr{C}} H_{\mathscr{C}} \cdot[U, U] \Rightarrow v u .
$$

Proposition 1.4. $\alpha_{U}$ is the unique natural transformation of bifunctors for which the diagram

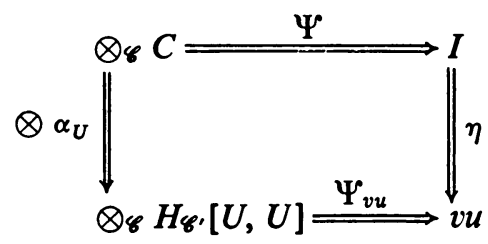

commutes.

Proof. This follows directly from 1.1.

Associated to $U$, we have two bifunctors $U^{*}$ and $U_{*}$ in $\left[\mathscr{C}^{\prime \circ} \times \mathscr{C}, \mathrm{Ab}\right]$ defined by $U^{*}=H_{\mathscr{C}},\left[U, \mathscr{C}^{\prime}\right]$ and $U_{*}=H^{\mathscr{C}}[U, \mathscr{C}]$. Since, for any object $C^{\prime}$ of $\mathscr{C}$,

$$
\mathscr{C}^{\prime}\left(\mathscr{C}^{\prime}, \quad\right) \otimes_{\mathscr{C}}, U^{*}=\left[U, \mathscr{C}^{\prime}\left(C^{\prime},\right)\right]_{\mathscr{C}},=v\left(\mathscr{C}^{\prime}\left(C^{\prime},\right)\right),
$$


$U^{*}$ is the bifunctor corresponding to $v$ and we have by 1.1 a natural transformation

$$
\Psi_{v}: \quad \otimes_{\mathscr{C}}, U^{*} \Rightarrow v .
$$

By 1.3 and the assumption $\otimes_{\mathscr{C}} U=u$, we have natural transformations

$$
u \Psi_{v}: \square \otimes_{\mathscr{C}},\left(U^{*} \otimes_{\mathscr{C}} U\right) \Rightarrow u v, \quad \Psi_{v} u: \quad \otimes_{\mathscr{C}}\left(U \otimes_{\mathscr{C}}, U^{*}\right) \Rightarrow v u .
$$

Therefore, by 1.1, we have the following:

Proposition 1.5. There is a unique natural transformation of bifunctors

$$
\beta_{U}: U^{*} \otimes_{\mathscr{C}} U \Rightarrow \mathscr{C}^{\prime}
$$

for which the diagram

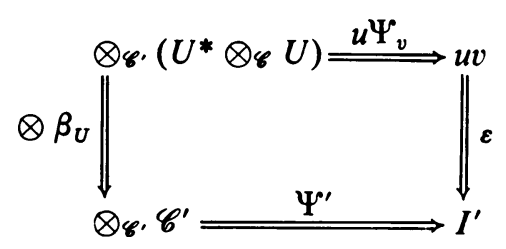

commutes.

PROPOSITION 1.6. There is a unique natural transformation of bifunctors

$$
\gamma_{U}: U \otimes_{\mathscr{C}}, U^{*} \Rightarrow H_{\mathscr{C}},[U, U]
$$

for which the diagram

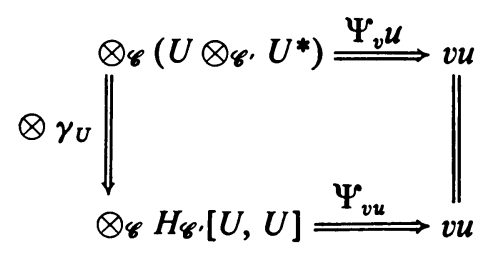

commutes.

Finally, we introduce a notion of "duality". To a bifunctor $U: \mathscr{C}^{\circ} \times \mathscr{C}^{\prime} \rightarrow \mathrm{Ab}$ we associate a bifunctor $U^{\circ}: \mathscr{C}^{\prime} \times \mathscr{C}^{\circ} \rightarrow \mathrm{Ab}$ given by $U^{\circ}\left(C^{\prime}, C\right)=U\left(C, C^{\prime}\right)$. This association trivially defines an equivalence of categories

$$
\left[\mathscr{C}^{\circ} \times \mathscr{C}^{\prime}, \mathrm{Ab}\right] \cong\left[\mathscr{C}^{\prime} \times \mathscr{C}^{\circ}, \mathrm{Ab}\right] .
$$

Using 1.2 and the fact that $\left(\mathscr{C}^{\prime \circ}\right)^{\circ}=\mathscr{C}^{\prime}$, we obtain an equivalence of categories

$$
\operatorname{Adj}\left(\left[\mathscr{C}^{\prime}, \mathrm{Ab}\right],[\mathscr{C}, \mathrm{Ab}]\right) \cong \operatorname{Adj}\left(\left[\mathscr{C}^{\circ}, \mathrm{Ab}\right],\left[\mathscr{C}^{\prime \circ}, \mathrm{Ab}\right]\right) .
$$

For $(v, u)$ an adjoint pair from $\left[\mathscr{C}^{\prime}, \mathrm{Ab}\right]$ to $[\mathscr{C}, \mathrm{Ab}]$, we let $\left(v^{\circ}, u^{\circ}\right)$ denote the corresponding adjoint pair, so that if the bifunctor $U$ corresponds to $(v, u)$, then $U^{\circ}$ corresponds to $\left(v^{\circ}, u^{\circ}\right)$. 
From the discussion of commutativity of the tensor product in [3], one may show that, for bifunctors $U: \mathscr{C}_{1}^{\circ} \times \mathscr{C}_{2} \rightarrow \mathrm{Ab}$ and $V: \mathscr{C}_{2}^{\circ} \times \mathscr{C}_{3} \rightarrow \mathrm{Ab}$,

$$
\left(U \otimes_{\mathscr{C}_{2}} V\right)^{\circ} \cong V^{\circ} \otimes_{\mathscr{C}_{2}^{\circ}} U^{\circ} .
$$

By direct calculation, one also shows that for bifunctors $U: \mathscr{C}_{1}^{\circ} \times \mathscr{C}_{2} \rightarrow \mathrm{Ab}$ and $V: \mathscr{C}_{1}^{\circ} \times \mathscr{C}_{3} \rightarrow \mathrm{Ab}$

$$
H^{\mathscr{C}_{1}}[U, V]=\left(H_{\mathscr{C}_{1}^{\circ}}\left[U^{\circ}, V^{\circ}\right]\right)^{\circ} \quad \text { and } \quad U_{*}=\left(U^{\circ *}\right)^{\circ} .
$$

The reader easily shows that the "duals" of $1.4,5$, and 6 hold.

2. Small projectives and generators. Recall that an object $P$ of a category $\mathscr{A}$ is called a small projective if $\mathscr{A}(P, \quad)$ is right continuous. (For example, if $R$ is a ring, the small projectives of ${ }_{R} \mathscr{M}$ are the finitely generated projective $R$-modules.)

Let $U: \mathscr{C}^{\circ} \times \mathscr{C}^{\prime} \rightarrow \mathrm{Ab}$ be a bifunctor. We shall say that $U$ is a small $\mathscr{C}^{\prime}$-projective if, for every object $A$ of $\mathscr{C}, U(A, \quad)$ is a small projective of [ $\left.\mathscr{C}^{\prime}, \mathrm{Ab}\right]$. One easily sees that this is equivalent to the functor $[U,]_{\mathscr{G}}$, being right continuous, so that by 1.1 one has the following:

Proposition 2.1. Let $U: \mathscr{C}^{\circ} \times \mathscr{C}^{\prime} \rightarrow \mathrm{Ab}$ be a bifunctor with corresponding adjoint pair $(v, u)$. Then $U$ is a small $\mathscr{C}^{\prime}$-projective if, and only if, $\Psi_{v}: \quad \otimes_{\mathscr{C}}, U^{*} \Rightarrow v$ is an isomorphism.

As an immediate consequence, we obtain the following:

THEOREM 2.2. Let $U: \mathscr{C}^{\circ} \times \mathscr{C}^{\prime} \rightarrow \mathrm{Ab}$ be a bifunctor with $(v, u)$ its associated adjoint pair. Suppose that $U$ is a small $\mathscr{C}^{\prime}$-projective. Then

(a) $v$ is right continuous and $U^{*}$ is its associated bifunctor;

(b) $v u$ is right continuous having both $U \otimes_{\mathscr{G}}, U^{*}$ and $H_{\mathscr{C}}[U, U]$ as associated bifunctors;

(c) $\gamma_{U}: U \otimes_{\mathscr{G}}, U^{*} \Rightarrow H_{\mathscr{C}},[U, U]$ (of 1.6) is an isomorphism.

Recall also that a collection of objects $\left\{D_{i}\right\}_{I}$ of a category $\mathscr{A}$ is called a generating family if, for any two objects $A$ and $B$ of $\mathscr{A}$ and for any two distinct morphisms $x_{1}, x_{2} \in \mathscr{A}(A, B)$, there is an $i \in I$ and a morphism $y: D_{i} \rightarrow A$ for which $x_{1} y \neq x_{2} y$.

Let $U: \mathscr{C}^{\circ} \times \mathscr{C}^{\prime} \rightarrow$ Ab be a bifunctor. We shall say that $U$ is a $\mathscr{C}^{\prime}$-generator if the collection of functors $\{U(A, \quad) \mid A$ an object of $\mathscr{C}\}$ is a generating family of $\left[\mathscr{C}^{\prime}, \mathrm{Ab}\right]$.

We prove the following:

THEOREM 2.3. Let $U: \mathscr{C}^{\circ} \times \mathscr{C}^{\prime} \rightarrow \mathrm{Ab}$ be a bifunctor and let $\beta_{U}: U^{*} \otimes_{\mathscr{C}} U \Rightarrow \mathscr{C}^{\prime}$ be the natural transformation of 1.5. Then

(a) if $U$ is a $\mathscr{C}^{\prime}$-generator, $\beta_{U}$ is an epimorphism;

(b) if there is a bifunctor $V: \mathscr{C}^{10} \times \mathscr{C} \rightarrow \mathrm{Ab}$ and a natural transformation of bifunctors $\nu: V \otimes_{\mathscr{C}} U \Rightarrow \mathscr{C}^{\prime}$ which is an epimorphism, then $U$ is a $\mathscr{C}^{\prime}$-generator. In particular, the converse of (a) holds. 
Proof. (a) Let $(v, u)$ be the adjoint pair associated to $U$, and recall the isomorphism $\theta$ corresponding to $(v, u)$ (discussed at the beginning of $\S 1$ ). Using $\theta$, we have for every object $B$ of $\mathscr{C}^{\prime}$ the following:

$$
\begin{aligned}
{\left[\left[U, \mathscr{C}^{\prime}(B, \quad)\right]_{\mathscr{C}^{\prime}} \otimes_{\mathscr{C}} U, \mathscr{C}^{\prime}(B, \quad)\right] } & \cong\left[u \mathscr{C}^{\prime}(B, \quad), \mathscr{C}^{\prime}(B, \quad)\right] \cong{ }^{\theta}\left[v \mathscr{C}^{\prime}(B, \quad), v \mathscr{C}^{\prime}(B, \quad)\right] \\
& \cong\left[\left[U, \mathscr{C}^{\prime}(B, \quad)\right]_{\mathscr{C}^{\prime}},\left[U, \mathscr{C}^{\prime}(B, \quad)\right] \mathscr{C}^{\prime}\right] .
\end{aligned}
$$

One easily shows, using 1.5 and the definition of the counit, that under this isomorphism $\beta_{U}(B, \quad)$ corresponds to the identity on $\left[U, \mathscr{C}^{\prime}(B, \quad)\right]_{\mathscr{C}^{\prime}}$.

Suppose $W: \mathscr{C}^{\prime \circ} \times \mathscr{C}^{\prime} \rightarrow \mathrm{Ab}$ is a bifunctor and $\lambda: \mathscr{C}^{\prime} \Rightarrow W$ is a nonzero natural transformation of bifunctors. Then there is an object $B$ of $\mathscr{C}^{\prime}$ so that $\lambda(B, \quad)$ : $\mathscr{C}^{\prime}(B, \quad) \Rightarrow W(B, \quad)$ is not zero. It follows easily, from the assumption that $U$ is a $\mathscr{C}^{\prime}$-generator, that the induced natural transformation

$$
\lambda(B,)_{*}:\left[U, \mathscr{C}^{\prime}(B, \quad)\right]_{\mathscr{C}^{\prime}} \Rightarrow[U, W(B, \quad)]_{\mathscr{C}^{\prime}}
$$

is not zero. In the commutative diagram induced by $\lambda(B, \quad)$,

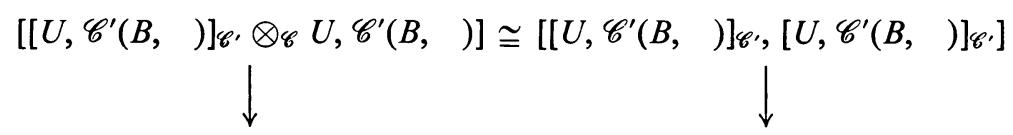

$\left[\left[U, \mathscr{C}^{\prime}(B, \quad)\right]_{\mathscr{C}^{\prime}} \otimes_{\mathscr{C}} U, W(B, \quad)\right] \cong\left[\left[U, \mathscr{C}^{\prime}(B, \quad)\right]_{\mathscr{C}^{\prime}},[U, W(B, \quad)]_{\mathscr{C}^{\prime}}\right]$,

since $\beta_{U}(B, \quad)$ corresponds to the identity in the upper row, $\lambda(B,) \beta_{U}(B, \quad)$ corresponds to $\lambda(B,)_{*}$ in the lower row. Consequently, $\lambda \beta_{U}$ is not zero, which shows that $\beta_{U}$ must be an epimorphism.

(b) Let $G, G^{\prime}$ be functors in $\left[\mathscr{C}^{\prime}, \mathrm{Ab}\right]$ and suppose $\lambda: G \Rightarrow G^{\prime}$ is a nonzero natural transformation. Then there is some object $B$ of $\mathscr{C}^{\prime}$ for which $\lambda_{B} \neq 0$, and consequently there is a natural transformation $x: \mathscr{C}^{\prime}(B, \quad) \Rightarrow G$ so that $\lambda x \neq 0$. Since $\nu(B, \quad): V(B, \quad) \otimes_{\mathscr{C}} U \Rightarrow C^{\prime}(B, \quad)$ is an epimorphism, $\lambda x \nu(B, \quad) \neq 0$.

Let $\left\{A_{i}\right\}_{I}$ be a collection of objects of $\mathscr{C}$ for which there is an epimorphism $\sum_{I} \mathscr{C}(A, \quad) \Rightarrow V(B, \quad)$. Therefore, $\left(\sum_{I} \mathscr{C}(A, \quad)\right) \otimes_{\mathscr{C}} U \Rightarrow V(B, \quad) \otimes_{\mathscr{C}} U$ is an epimorphism, so that the composition of this natural transformation with $\lambda x \nu(B, \quad)$ is nonzero. But $\left(\sum_{I} \mathscr{C}(A),\right) \otimes_{\mathscr{C}} U \cong \sum_{I} U\left(A_{i}, \quad\right)$. It follows that for some $i \in I$, there is a natural transformation $y: U\left(A_{i}, \quad\right) \Rightarrow G$ so that $\lambda y \neq 0$. Therefore, $U$ is a $\mathscr{C}^{\prime}$-generator.

3. Morita context. In this section, we generalize to bifunctors the notion of a "Morita context" given in [2] for bimodules.

Suppose $\mathscr{C}, \mathscr{C}^{\prime}$ are small categories, $U: \mathscr{C}^{\circ} \times \mathscr{C}^{\prime} \rightarrow \mathrm{Ab}$ and $V: \mathscr{C}^{\prime \circ} \times \mathscr{C} \rightarrow$ Ab are bifunctors, and $\mu: U \otimes_{\mathscr{C}}, V \Rightarrow \mathscr{C}$ and $\nu: V \otimes_{\mathscr{C}} U \Rightarrow \mathscr{C}^{\prime}$ are natural transformations. We shall say that $(U, V, \mu, \nu)$ forms a Morita context if and only if the diagrams
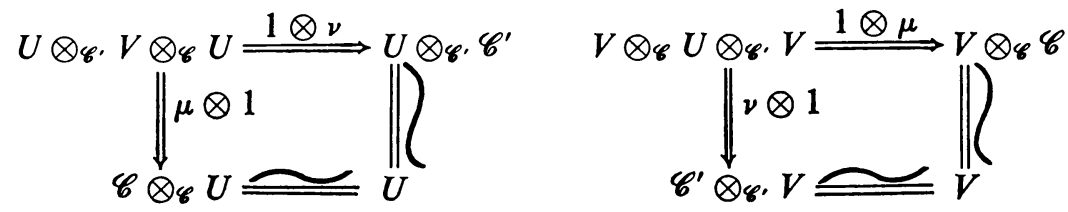
commute. One notes that if $(U, V, \mu, \nu)$ forms a Morita context, so do $(V, U, \nu, \mu)$ and $\left(U^{\circ}, V^{\circ}, \mu^{\circ}, \nu^{\circ}\right)$.

Suppose $u:[\mathscr{C}, \mathrm{Ab}] \rightarrow\left[\mathscr{C}^{\prime}, \mathrm{Ab}\right]$ and $v:\left[\mathscr{C}^{\prime}, \mathrm{Ab}\right] \rightarrow[\mathscr{C}, \mathrm{Ab}]$ are functors which define an equivalence of categories. Then, in particular, both functors are right continuous, so that by 1.1 there are bifunctors $U: \mathscr{C}^{\circ} \times \mathscr{C}^{\prime} \rightarrow \mathrm{Ab}$ and $V: \mathscr{C}^{10} \times \mathscr{C}$ $\rightarrow \mathrm{Ab}$ and isomorphisms $\Psi_{U}: \otimes_{\mathscr{C}} U \Rightarrow u$ and $\Psi_{V}: \otimes_{\mathscr{C}}, V \Rightarrow v$. By 1.3, we have isomorphisms $\Psi_{1}: \quad \otimes_{\mathscr{C}}\left(U \otimes_{\mathscr{C}}, V\right) \Rightarrow v u$ and $\Psi_{2}: \quad \otimes_{\mathscr{C}},\left(V \otimes_{\mathscr{C}} U\right) \Rightarrow u v$, and the inverse $\eta^{-1}$ of the unit of the adjoint pair $(v, u)$ and the counit $\varepsilon$ induce unique natural transformations $\mu: U \otimes_{\mathscr{C}}, V \Rightarrow \mathscr{C}$ and $\nu: V \otimes_{\mathscr{C}} U \Rightarrow \mathscr{C}^{\prime}$ for which the diagrams
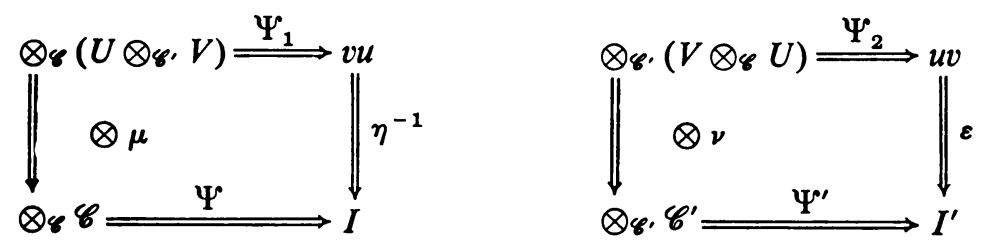

commute.

THEOREM 3.1. Let $(v, u)$ be an adjoint pair in $\operatorname{Adj}\left(\left[\mathscr{C}^{\prime}, \mathrm{Ab}\right],[\mathscr{C}, \mathrm{Ab}]\right)$ which defines an equivalence of categories, let $U: \mathscr{C}^{\circ} \times \mathscr{C}^{\prime} \rightarrow \mathrm{Ab}$ and $V: \mathscr{C}^{\prime \circ} \times \mathscr{C} \rightarrow \mathrm{Ab}$ be the bifunctors associated to $U$ and $V$ respectively, and let $\mu: U \otimes_{\mathscr{C}}, V \Rightarrow \mathscr{C}$ and $\nu: V \otimes_{\mathscr{C}} U \Rightarrow \mathscr{C}^{\prime}$ be the natural transformations induced by the inverse of the unit $\eta^{-1}$ and by the counit $\varepsilon$ of the adjoint pair $(v, u)$ respectively. Then $(U, V, \mu, \nu)$ forms a Morita context.

Proof. The identities $(*)$ induced identities

$$
\begin{aligned}
& \left(U \cong C \otimes_{\mathscr{C}} U \stackrel{\mu^{-1} \otimes 1}{=} U \otimes_{\mathscr{C}}, V \otimes_{\mathscr{C}} U \stackrel{1 \otimes_{\nu}}{=} U \otimes_{\mathscr{C}}, \mathscr{C}^{\prime} \cong U\right)=1_{U}, \\
& \left(V \cong V \otimes_{\mathscr{C}} \mathscr{C} \stackrel{1 \otimes^{-1}}{=} V \otimes_{\mathscr{C}} U \otimes_{\mathscr{C}} V \stackrel{\nu \otimes_{1}}{=} \mathscr{C}^{\prime} \otimes_{\mathscr{C}}, V \cong V\right)=1_{V}
\end{aligned}
$$

which in turn show that the diagrams $(* *)$ commute.

The following theorem, which generalizes "Morita 1" of [1], provides a converse to 3.1 .

Theorem 3.2. Let $\mathscr{C}, \mathscr{C}^{\prime}$ be categories, $U: \mathscr{C}^{\circ} \times \mathscr{C}^{\prime} \rightarrow \mathrm{Ab}$ and $V: \mathscr{C}^{\prime \circ} \times \mathscr{C} \rightarrow \mathrm{Ab}$ be bifunctors, and $\mu: U \otimes_{\mathscr{C}}, V \Rightarrow \mathscr{C}$ and $\nu: V \otimes_{\mathscr{C}} U \Rightarrow \mathscr{C}^{\prime}$ be natural transformations. Suppose $(U, V, \mu, \nu)$ forms a Morita context and that $\mu$ and $\nu$ are epimorphisms. Then

(a) $\mu$ and $\nu$ are isomorphisms,

(b) the pair $(v, u)$, with

$$
u=\otimes_{\mathscr{C}} U:[\mathscr{C}, \mathrm{Ab}] \rightarrow\left[\mathscr{C}^{\prime}, \mathrm{Ab}\right] \text { and } v=\otimes_{\mathscr{C}}, V:\left[\mathscr{C}^{\prime}, \mathrm{Ab}\right] \rightarrow[\mathscr{C}, \mathrm{Ab}],
$$

defines an equivalence of categories, 
(c) $U$ is a small $\mathscr{C}^{\prime}$-projective and a $\mathscr{C}^{\prime}$-generator, and $V$ is a small $\mathscr{C}$-projective and a $\mathscr{C}$-generator,

(d) $V \cong U^{*} \cong U_{*}$,

(e) the natural transformations

$$
\alpha_{U}: \mathscr{C} \Rightarrow H_{\mathscr{C}}[U, U] \text { and } \alpha_{V}: \mathscr{C}^{\prime} \Rightarrow H_{\mathscr{C}}[V, V]
$$

of 1.4 are isomorphisms.

Furthermore, $\left(U^{\circ}, V^{\circ}, \mu^{\circ}, \nu^{\circ}\right)$ forms a Morita context with $\mu^{\circ}$ and $\nu^{\circ}$ epimorphisms, so that $(\mathrm{a})^{\circ}-(\mathrm{e})^{\circ}$ hold.

Since $(V, U, \nu, \mu)$ and $\left(U^{\circ}, V^{\circ}, \mu^{\circ}, \nu^{\circ}\right)$ form Morita contexts and the dual of an epimorphism of bifunctors is an epimorphism, we need only to show (a)-(e) for $U$ and $\mu$.

We first prove the following:

Lemma 3.3. Let $W: \mathscr{C}^{\circ} \times \mathscr{C} \rightarrow \mathrm{Ab}$ be a bifunctor and $\tau: W \Rightarrow \mathscr{C}$ a natural transformation for which the diagram

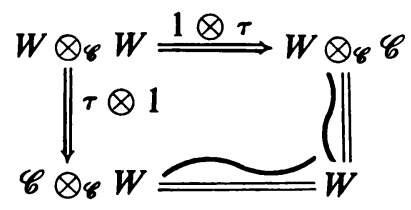

commutes. Then if $\tau$ is an epimorphism, $\tau$ is an isomorphism.

Proof of lemma. Suppose $K: \mathscr{C}^{\circ} \times \mathscr{C} \rightarrow \mathrm{Ab}$ is a bifunctor and $\lambda: K \Rightarrow W$ is a natural transformation for which $\tau \lambda=0$. It follows from the commutivity of (***) that the diagram

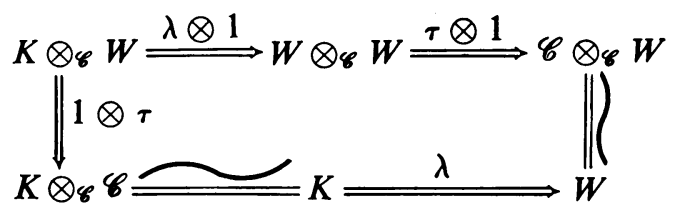

commutes. Since the top row is zero, it follows that, if $\tau$ is an epimorphism, $1 \otimes \tau$ is an epimorphism, so that $\lambda=0$, and consequently $\tau$ is a monomorphism also.

Proof of theorem. (a) follows easily from the Lemma by letting $U \otimes_{\mathscr{C}}, V=W$ and $\mu=\tau$. One easily sees that the commutivity of the diagrams of $(* *)$ implies that of $(* * *)$.

(b) follows easily from (a).

(c) It follows from (b) that $(v, u)$ is an adjoint pair, so that $v \cong[U,]_{C^{\prime}}$. Since $v$ is right continuous, $U$ is a small $\mathscr{C}^{\prime}$-projective. Since $\nu$ is an epimorphism, $U$ is a $\mathscr{C}^{\prime}$-generator by $2.3(\mathrm{~b})$. 
(d) By (c) and 2.2, $U^{*}$ is associated to $v$. But $V$ is also, so that there is a canonical isomorphism $\lambda: V \cong U^{*}$ for which the diagram

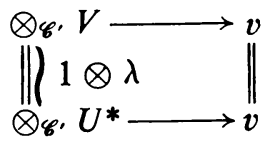

commutes. Dually, one has $V^{\circ} \cong\left(U^{\circ}\right)^{*}=\left(U_{*}\right)^{\circ}$, so that $V \cong U_{*}$.

(e) From (d) we have a canonical isomorphism $\lambda: V \cong U^{*}$ induced by the identity on $v$. Using the characterizations of $\alpha_{U}$ and $\gamma_{U}$ given in 1.4 and 1.6 respectively, one easily shows that $\alpha_{U}=\gamma_{U}(1 \otimes \lambda) \nu^{-1}$. Since $\nu$ is an isomorphism by (a), $1 \otimes \lambda$ is an isomorphism, and $\gamma_{U}$ is an isomorphism by (c) and 2.2(c), $\alpha_{U}$ is an isomorphism.

We remark that the other statements of [2, Morita 1] are easily generalized and proved also.

4. Progenerators and equivalences of categories. Let $U: \mathscr{C}^{\circ} \times \mathscr{C}^{\prime} \rightarrow \mathrm{Ab}$ be a bifunctor. We shall say that $U$ is a $\mathscr{C}-\mathscr{C}^{\prime}$ progenerator if $U$ is a small $\mathscr{C}^{\prime}$-projective $\mathscr{C}^{\prime}$-generator and $\alpha_{U}: \mathscr{C} \Rightarrow H_{\mathscr{C}},[U, U]$ is an isomorphism. (This generalizes the definition of an $R-S$ progenerator given in [2].) In this section we show that these are precisely the bifunctors whose associated adjoint pairs define equivalences of categories. In fact, we have the following:

THEOREM 4.1. Let $U: \mathscr{C}^{\circ} \times \mathscr{C}^{\prime} \rightarrow \mathrm{Ab}$ be a bifunctor, and let $(v, u)$ be its associated adjoint pair in $\operatorname{Adj}\left(\left[\mathscr{C}^{\prime}, \mathrm{Ab}\right],[\mathscr{C}, \mathrm{Ab}]\right)$. Then the following are equivalent:

(a) $(v, u)$ defines an equivalence of categories;

(b) $U$ is a $\mathscr{C}-\mathscr{C}^{\prime}$ progenerator;

(c) $\left(v^{\circ}, u^{\circ}\right)$ defines an equivalence of categories;

(d) $U^{\circ}$ is a $\mathscr{C}^{\prime \circ}{ }^{\circ} \mathscr{C}^{\circ}$ progenerator.

Proof. One easily shows, using 3.1 and 3.2 , that (a) $\Rightarrow$ (b) and (a) $\Rightarrow$ (c) and, by duality, (c) $\Rightarrow$ (d) and (c) $\Rightarrow$ (a).

Suppose $U$ is a $\mathscr{C}-\mathscr{C}^{\prime}$ progenerator and let $V=U^{*}$. Since $U$ is a small $\mathscr{C}^{\prime}$-projective, we have by 2.2 that $V$ is associated to $v$ and that $\gamma_{U}: U \otimes_{\mathscr{C}}, V \Rightarrow H_{\mathscr{C}},[U, U]$ is an isomorphism. Since $\alpha_{U}: \mathscr{C} \Rightarrow H_{\mathscr{C}}[U, U]$ is assumed to be an isomorphism, $\mu: U \otimes_{\mathscr{C}}, V \Rightarrow \mathscr{C}$, defined by $\mu=\alpha_{U}^{-1} \gamma_{U}$, is an isomorphism. Since $U$ is a $\mathscr{C}^{\prime}$-generator, $\nu: V \otimes_{\mathscr{C}} U \Rightarrow \mathscr{C}^{\prime}$, defined by $\nu=\beta_{U}$, is an epimorphism by 2.3(a). Using 1.4, 1.5 , and 1.6 , one easily shows that the identities $(*)$ induce the identities

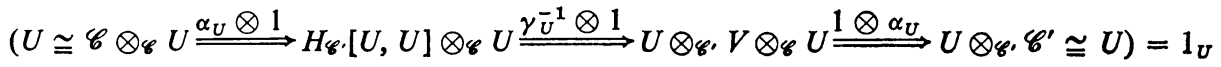

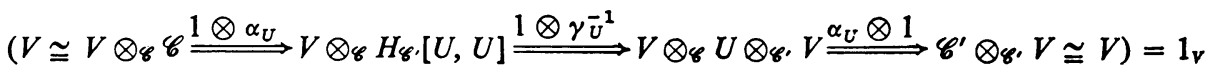


so that $(U, V, \mu, \nu)$ forms a Morita context (i.e. the diagrams of (**) commute). Furthermore, $(U, V, \mu, \nu)$ satisfies the hypotheses of 3.2 , so that by $3.2(\mathrm{~b}),(v, u)$ defines an equivalence of categories. Thus, (b) $\Rightarrow$ (a) and dually (d) $\Rightarrow$ (c).

CoRollary 4.2. If $U_{1}: \mathscr{C}_{1}^{\circ} \times \mathscr{C}_{2} \rightarrow \mathrm{Ab}$ is a $\mathscr{C}_{1}-\mathscr{C}_{2}$ progenerator and $U_{2}: \mathscr{C}_{2}^{\circ} \times \mathscr{C}_{3}$ $\rightarrow \mathrm{Ab}$ is a $\mathscr{C}_{2}-\mathscr{C}_{3}$ progenerator, then $U_{1} \otimes \mathscr{C}_{2} U_{2}$ is a $\mathscr{C}_{1}-\mathscr{C}_{3}$ progenerator.

Proof. This follows from 1.3, 4.1, and the fact that composition of equivalences of categories is an equivalence of categories.

For a category $\mathscr{A}$, let Aut $(\mathscr{A})$ denote the isomorphism classes of functors $u: \mathscr{A} \rightarrow \mathscr{A}$ for which there is a $v: \mathscr{A} \rightarrow \mathscr{A}$ such that $(v, u)$ defines an equivalence of categories. Clearly Aut $(\mathscr{A})$ is a group under composition.

COROLlaRY 4.3. Let $\mathscr{C}$ be a small category. Then the isomorphism classes of $\mathscr{C}-\mathscr{C}$ progenerators form a group under tensor products of functors and the assignment $U \mapsto \otimes_{\mathscr{C}} U$ defines a group isomorphism from this group to Aut ([ $\left.\left.\mathscr{C}, \mathrm{Ab}\right]\right)$.

Proof. This is clear, once we note that the inverse of $U$ is $U^{*}$.

\section{REFERENCES}

1. H. Bass, Algebraic K-theory, Benjamin, New York, 1968. MR 40 \#2736.

2. —_ The Morita theorems, Mimeographed Notes, University of Oregon, Eugene, Oregon.

3. J. I. Fisher, The tensor product of functors; satellites; and derived functors, J. Algebra 8 (1968), 277-294. MR 38 \#5874.

4. J. F. Palmquist and D. C. Newell, Bifunctors and adjoint pairs, Trans. Amer. Math. Soc. 155 (1971), 293-303.

5. S. Mac Lane, Categorical algebra, Bull. Amer. Math. Soc. 71 (1965), 40-106. MR 30 \#2053.

Department of Mathematics, University of California, Irvine, California 92664 